\title{
Consequences of Unit Cell Design in Metamaterial Perfect Absorbers
}

\author{
F. BAGCI AND B. AKAOGLU* \\ Department of Engineering Physics, Faculty of Engineering, Ankara University, 06100 Besevler, Ankara, Turkey
}

\begin{abstract}
Metamaterials are a new class of composite materials with unusual properties that allow controlling of electromagnetic waves by properly engineering the response functions, which are not observed in constituent materials. However, since absorption of metamaterials is mainly based on electromagnetic resonances, the operating bandwidth is relatively narrow. Utilization of more than a single metallic structure with different geometrical parameters in each unit cell is a common way of accomplishing multiple band and/or broadband absorption. There are two usual approaches for this purpose: (a) multilayer unit cell design where metallic structures on dielectric substrate are stacked one on top of the other; (b) side by side unit cell design where metallic structures are distributed on a dielectric substrate. However, to the best of our knowledge, these two different approaches are not comparatively investigated. In this study, we propose metamaterial-based perfect absorbers with two different unit cell designs and simulate transmittances, reflectances and absorbances for each design by a commercial electromagnetic solver, CST Microwave Studio. It is found that each design has its own advantages in terms of device thickness, absorption bandwidth and angular dependence, which might be severely important for particular purposes.
\end{abstract}

DOI: 10.12693/APhysPolA.129.792

PACS/topics: 81.05.Xj, 41.20.Jb

\section{Introduction}

The phenomenon of artificially engineered materials was first offered by Veselago theoretically in 1968 [1] and demonstrated experimentally in 2000 by using split ring resonators (SRRs) and continuous wires [2]. In these structures thin wires exhibit an electric resonance below their plasma frequency, whereas SRRs exhibit a magnetic resonance. However, metamaterials (MMs) that are composed of SRRs and thin wires have several restrictions, such as difficulty in utilization of the SRRs at optical frequencies and difficulty of alignment of the multiple layers [3]. These restrictions are overcome by the use of planar MMs, of which the propagation direction is normal to the MM surface [4].

MMs find great applications as absorbers in areas, such as sensing [5], solar energy absorption [6], wireless communication [7]. Moreover, they can be used in military applications to improve the performance of radars and provide concealment against the radar systems [8]. Initially, SRRs were proposed as the microwave absorbers but later were replaced with electric field-driven LC (ELC) resonators, which are planar MMs with strongly uniform electric field coupled elements [9].

Besides having a high level of absorption, it is desirable for the MM absorbers (MAs) to have a broad bandwidth absorption performance in small geometric dimensions. Several approaches have been demonstrated to achieve this purpose. The first approach utilizes multiple elements resonating at different frequencies, incorporated in one unit cell $[10,11]$. The second approach uses stacked multiple layers in which all layers have the same ground

*corresponding author; e-mail: akaoglu@eng.ankara.edu.tr plane $[12,13]$, however the fabrication of such MM can be difficult. For example, Ding et al. proposed a MA which is composed of 20 metal patches that are separated by dielectric layers and arranged in a pyramid form with their widths tapered linearly [13]. Alternatively, different sections of a single structure can be designed to resonate at different frequencies in order to obtain a multiband MA [14]. However, in these designs it is necessary to avoid coupling between different resonators since they share a section of the resonator. Lastly, lumped elements can be integrated into the MMs to extend the bandwidth or to achieve multiband operation [15]. Although lumped elements may offer switchable operating frequencies, the feasibility of incorporation of these elements into small resonators can be problematic, especially at higher frequencies.

The MA with near unity absorption at a specific microwave frequency is obtained by equalizing the permittivity and permeability of a MM close to the free space impedance [16]. However, the FWHM bandwidth of that structure is limited to a fractional bandwidth of $0.4 \%$ around the center resonance frequency due to the intrinsic resonance of the MM. In this study it is demonstrated that the FWHM bandwidths of single MMs can be increased by using two MM structures with different geometrical parameters. The performance of the new designs is compared with that of the conventional single MM design in terms of absorption frequency, absorption bandwidth and incidence angle variation. It is shown that the proposed designs are capable to operate at wide variations of the angle of the incident wave.

Two different approaches are used for the bandwidth enlargement: (a) multilayer unit cell design where MMs are stacked one on top of the other; (b) side by side design where MMs are arranged in a row. Although similar approaches are utilized by different groups in different 
studies as aforementioned, to the best of our knowledge, these methods are not comparatively investigated. We found that each approach has its own advantages in terms of device thickness, absorption bandwidth and angular dependence, which might be severely important for various shielding applications.

\section{Design and simulation}

In this paper the MA structure introduced in [16] is studied in depth to extend the bandwidth of the absorber. Two MM structures, one as reference and the other having slightly different optimized parameters are used for this purpose. The MM structures consist of a square shaped electric resonator at the front face and a metal cut-wire at the back face which lie on $0.4 \mathrm{~mm}$ thick FR-4 substrates with a small air gap of $0.25 \mathrm{~mm}$ between them. The dimensions of the reference MM are as follows in millimeters: $a 1=4.2, a 2=12, w 1=3.9, G=0.61$ and $t 1=0.6$. The back side of the substrate consists of a metal cut-wire with a width of $1.7 \mathrm{~mm}$ and a height of $11.8 \mathrm{~mm}$ at the center. Two MM structures are used in stacked or side-by-side configuration in the analysis, as shown in Fig. 1a and 1b, respectively.

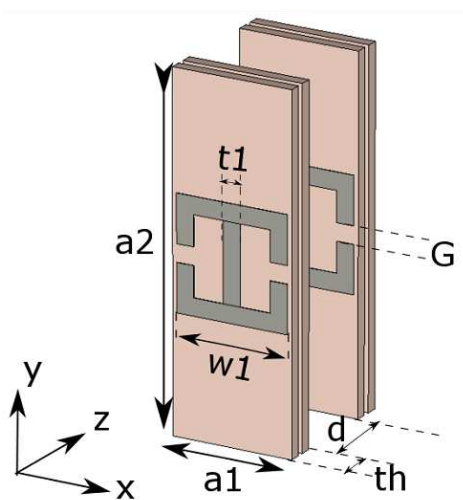

(a)

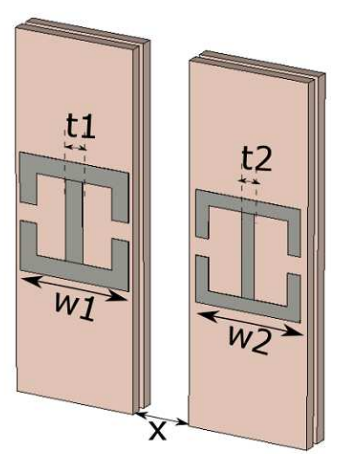

(b)
Fig. 1. MA design based on (a) stacked, (b) side-byside configuration.

The simulation of the structures is performed by using a commercial full wave electromagnetic solver (CST Microwave Studio). The metal is considered as a perfect electric conductor. The dielectric constant and losstangent of the FR-4 substrate are taken as 4.3 and 0.025 , respectively. Periodic boundary conditions are imposed on the $x$ and $y$ axes, whereas open boundaries are used in the propagation direction with Floquet ports. A plane wave is sent normally through the structure with the electric field $y$-polarized and magnetic field $x$-polarized. Optimization is performed for the $t 2$ and $w 2$ parameters of the introduced MM structure, including the distance $x$ or $d$ between the MMs and the unit cell dimension along the $x$-axis $\left(a_{x}\right)$. The other geometric parameters of the introduced MM are taken the same as those of the reference MM. In the parametric scan $t 2$ is changed from 0.4 to $0.6 \mathrm{~mm}, d$ or $x$ are changed from 0 to $3 \mathrm{~mm}, a_{x}$ is changed from 4.2 to $7.2 \mathrm{~mm}$ for the stacked configuration or from 8.4 to $14.4 \mathrm{~mm}$ for the side-by-side configuration by $0.05 \mathrm{~mm}$ steps; $w 2$ is changed from 3.5 to $3.9 \mathrm{~mm}$ by $0.1 \mathrm{~mm}$ steps, simultaneously.

The absorption ratio with respect to the frequency is calculated as $A(\omega)=1-R(\omega)-T(\omega)$, where $A(\omega)$, $R(\omega)$ and $T(\omega)$ represent the absorbance, reflectance and transmittance, respectively. Since the bottom plane has a long cut-wire, the transmission was low and the minimization of the reflectance in a large bandwidth was more crucial in our analysis.

\section{Results and discussion \\ 3.1. Metamaterial absorber based on stacked configuration}

A broadband absorption response can be obtained if the resonance frequencies of the elements are close to each other. The widest bandwidth is obtained from the parametric scan for $t 2=0.4 \mathrm{~mm}, w 2=3.6 \mathrm{~mm}, d=1.5 \mathrm{~mm}$ and $a_{x}=5.2 \mathrm{~mm}$. As shown in Fig. 2, the FWHM bandwidth of the reference MM (black curve) is $0.42 \mathrm{GHz}$ with a center frequency of $10.38 \mathrm{GHz}$ and the FWHM bandwidth of the other MM is $0.35 \mathrm{GHz}$ with a center frequency of $10.16 \mathrm{GHz}$. When the MMs are combined in a unit cell, the FWHM absorbance bandwidth is $0.58 \mathrm{GHz}$ with a relative FWHM bandwidth of $5.45 \%$. The FWHM bandwidth is increased $37.35 \%$ according to that of the reference and $64.12 \%$ according to the introduced MM. Moreover, the absorption level of $97 \%$ is reached when the MMs are combined.

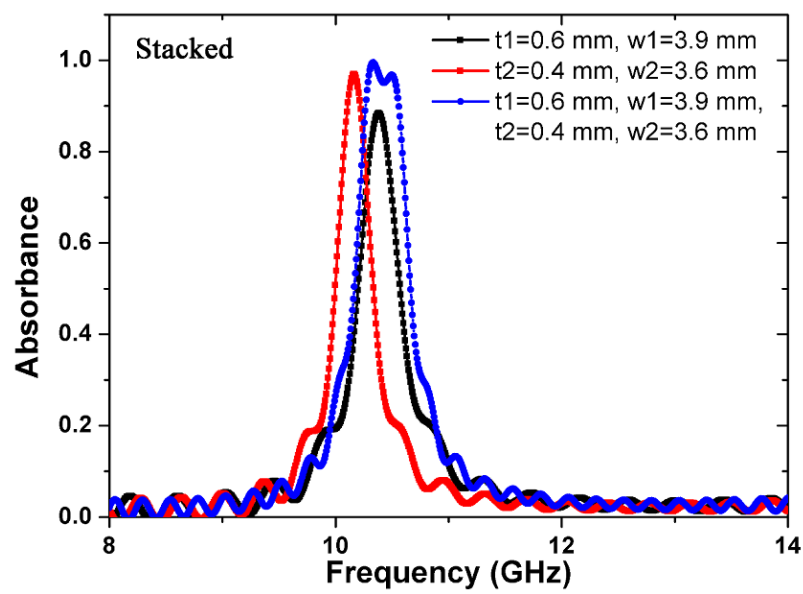

Fig. 2. Absorption spectra of the individual MMs and their combination in stacked configuration.

Since an electromagnetic wave can impinge onto the structure from any direction, it is quite necessary to investigate the absorbance behaviour for varying incident angles. The absorbance spectrum related to the incidence angle is shown in Fig. 3a and the variations of 

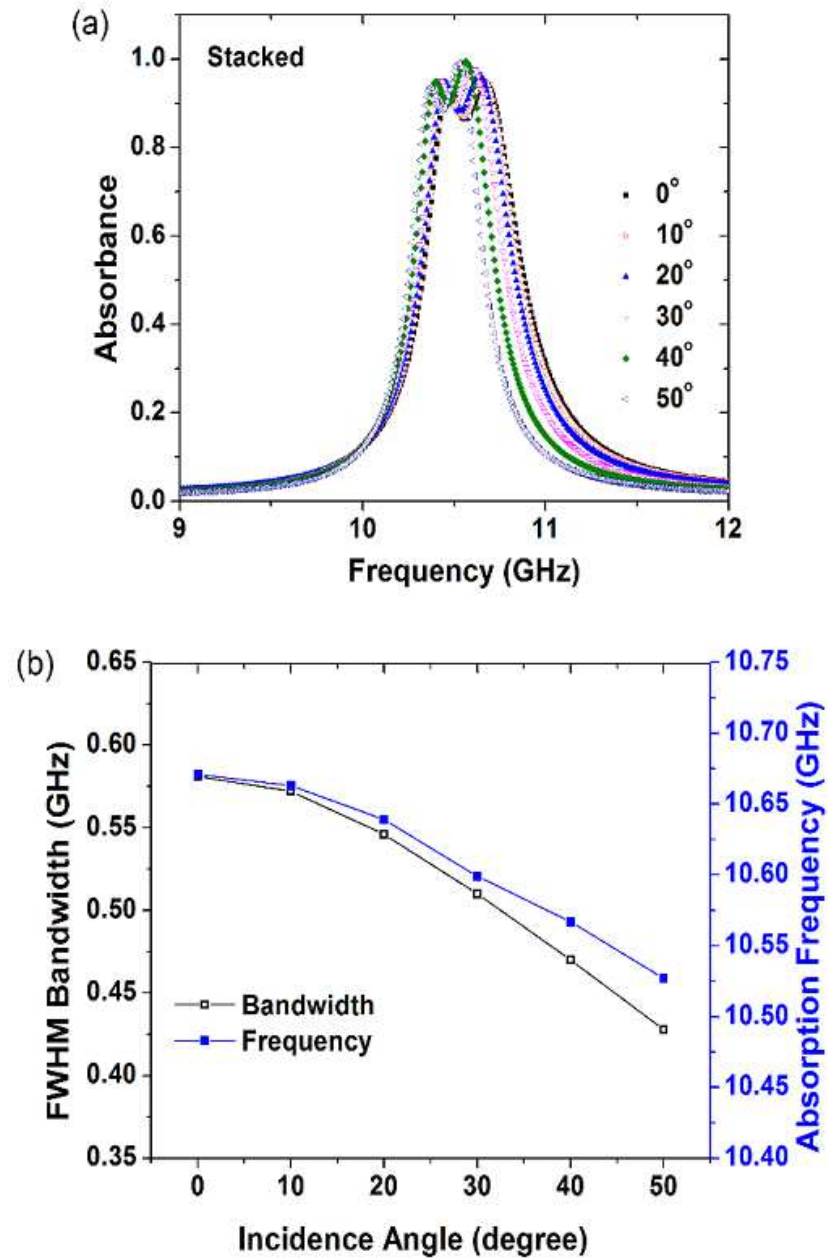

Fig. 3. Angular dependence of the (a) absorption spectrum, (b) absorption frequency and bandwidth of the stacked configuration.

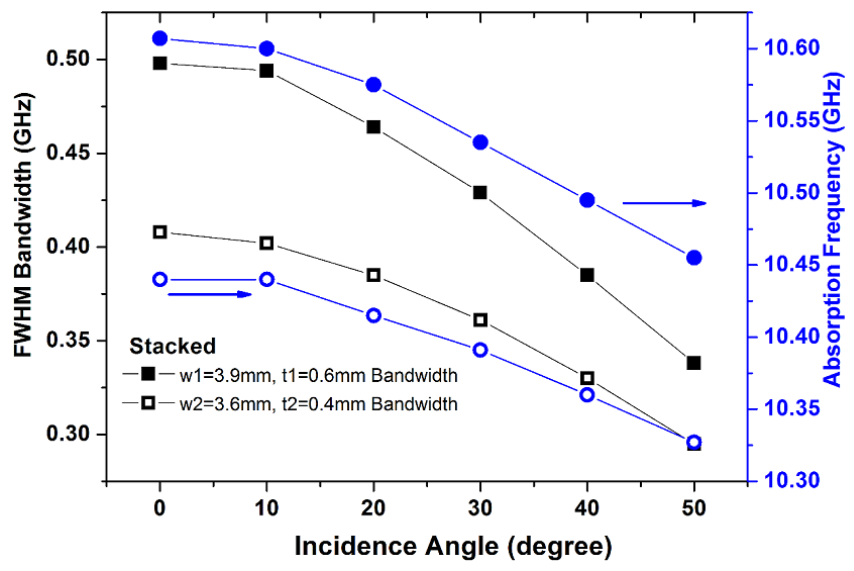

Fig. 4. Angular dependence of the absorption frequency and bandwidth of the individual MMs composing the stacked configuration. the absorption frequency and FWHM in terms of incidence angle are given in Fig. 3b for the stacked configuration. The maximum absorption frequency decreases slightly from $10.67 \mathrm{GHz}$ to $10.53 \mathrm{GHz}$ and the FWHM bandwidth decreases from $0.58 \mathrm{GHz}$ to $0.43 \mathrm{GHz}$ as the incidence angle increases from $0^{\circ}$ to $50^{\circ}$. Besides, the absorption level shows negligible variation against incidence angle variation.

The dependence of the absorption frequency and bandwidth on the incident angle is also examined for the individual MMs (Fig. 4) in order to compare it with the performance of the stacked configuration. The air gap at the left and right side of the unit cell is kept the same in the simulations. It is found that the absorption frequency and FWHM bandwidth do not change more than those for the individual MMs under oblique incidence.

\subsection{Metamaterial absorber \\ based on side-by-side configuration}

The optimization was also performed for the side-byside configuration to compare the absorption efficiency with that of the stacked configuration. The widest absorption band is achieved for $t 2=0.45 \mathrm{~mm}, w 2=$ $3.7 \mathrm{~mm}, x=2 \mathrm{~mm}$ and $a_{x}=14.4 \mathrm{~mm}$. The FWHM absorbance bandwidth of the reference MM is (black curve) $0.42 \mathrm{GHz}$ with a center frequency of $10.38 \mathrm{GHz}$ and the FWHM bandwidth of the other MM is $0.384 \mathrm{GHz}$ with a center frequency of $10.33 \mathrm{GHz}$. When the MMs are combined in a unit cell, the FWHM absorbance bandwidth is $0.50 \mathrm{GHz}$ with a relative FWHM bandwidth of $4.8 \%$. The FWHM bandwidth is increased $19.15 \%$ according to that of the reference and $31.25 \%$ according to the introduced MM structure (Fig. 5). The maximum absorbance level of the side-by-side configuration is $93 \%$ which is a little lower, compared to the stacked configuration.

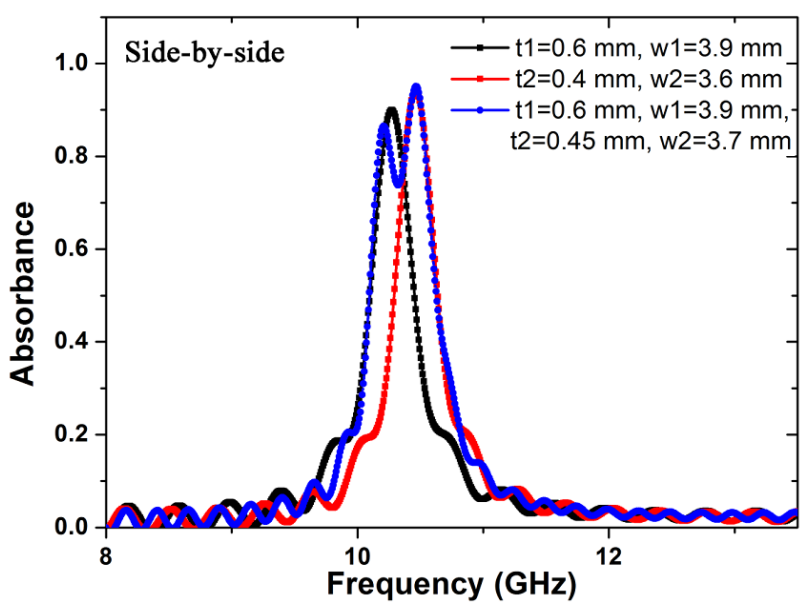

Fig. 5. Absorption spectra of the individual MMs and their combination in side-by-side configuration.

The dependence of the absorption spectrum of the sideby-side configuration on the incidence angle is shown 
in Fig. 6. The maximum absorption frequency decreases slightly from $10.48 \mathrm{GHz}$ to $10.44 \mathrm{GHz}$ and the FWHM bandwidth decreases from $0.50 \mathrm{GHz}$ to $0.37 \mathrm{GHz}$ as the incidence angle increases from $0^{\circ}$ to $50^{\circ}$, as shown in Fig. 6a and 6b, respectively. In fact, the FWHM bandwidth is $0.46 \mathrm{GHz}$ at $40^{\circ}$, so it can be deduced that the FWHM bandwidth is maintained up to $40^{\circ}$ incident angle. Although the absorption level of the side-by-side configuration is a little lower, the variation of the frequency against incident angle is much smaller for this configuration than for the stacked configuration.
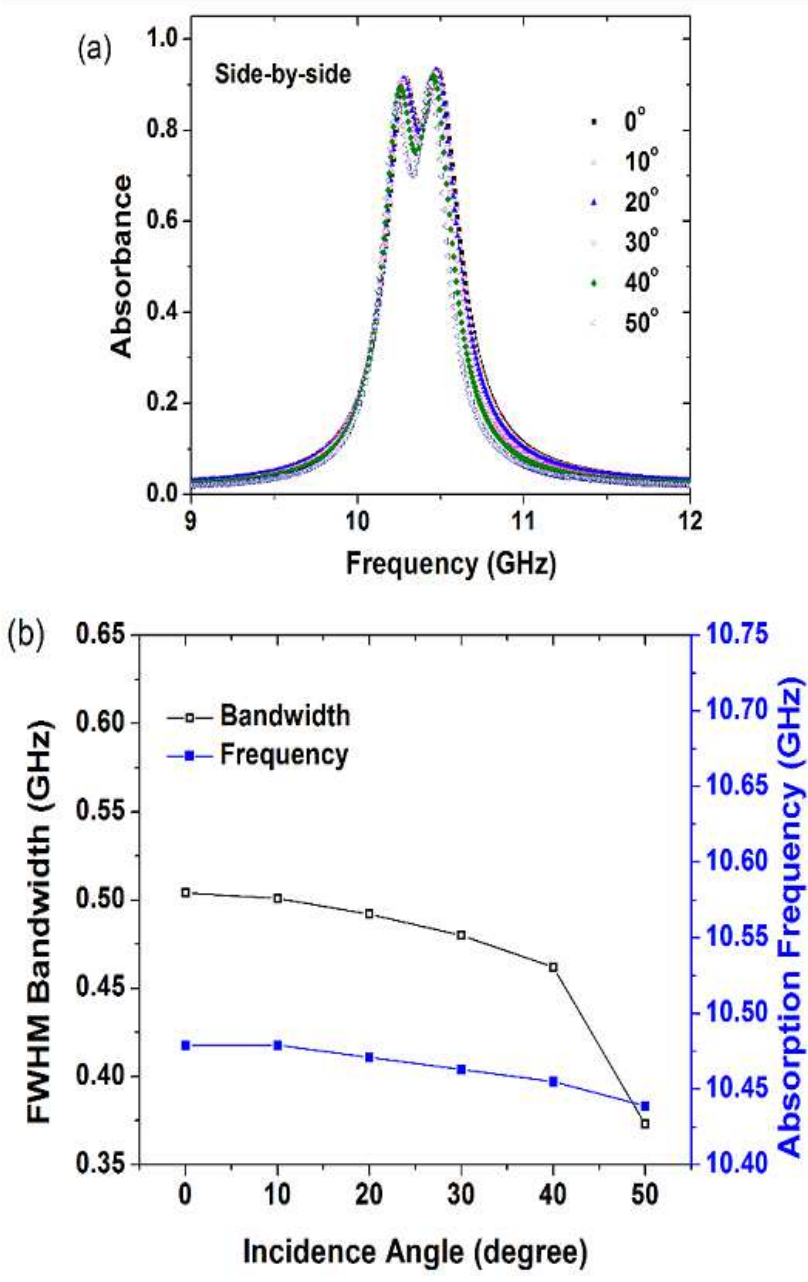

Fig. 6. Angular dependence of the (a) absorption spectrum, (b) absorption frequency and bandwidth of the side-by-side configuration (same scales are used in (b) as in Fig. 3b).

The absorption frequency and bandwidth of the individual MMs against incidence angle variation are illustrated in Fig. 7. The absorption frequency and bandwidth change by approximately the same amount for the side-by-side configuration and for the single MM structures, which indicates that the absorption bandwidth improves while preserving the absorption performance under incident angle variation.

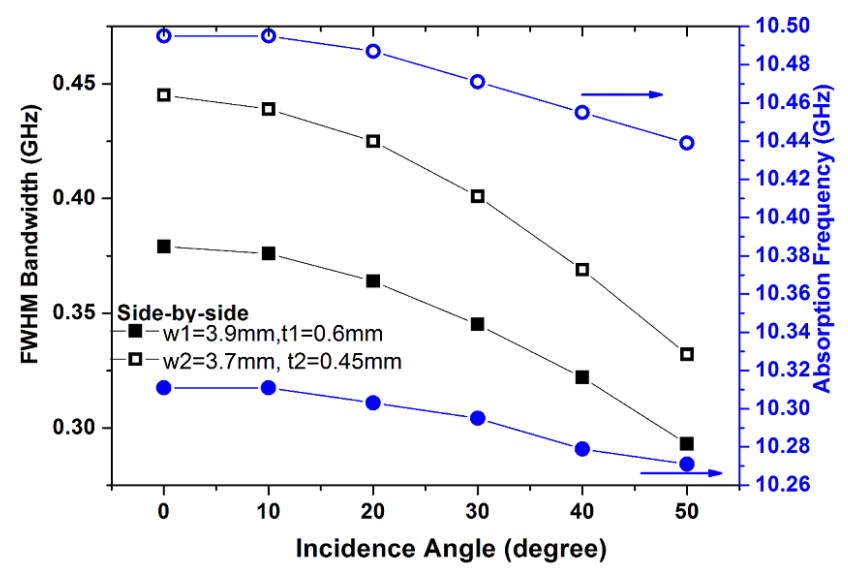

Fig. 7. Angular dependence of the absorption frequency and bandwidth of the individual MMs composing the side-by-side configuration.

\section{Conclusions}

In this study a MA based on a stacked or side-byside configuration is proposed by utilizing two MMs, one of which is optimized to extend the bandwidth of the inherently narrow-band MA. When the two MMs are combined in a unit cell structure, the bandwidth of the absorber is enlarged at nearly the same absorption frequency. Stacked configuration shows wider FWHM absorption bandwidth and higher level of absorption, which may be due to a destructive interference of multiple reflections in the gap region between MMs. However, the stacked configuration has a thickness of $0.127 \lambda$, whereas the side-by-side configuration is only $0.036 \lambda$ thick ( $\lambda$ in vacuum). The absorption frequency and bandwidth change by a smaller amount for the side-by-side configuration than for the stacked configuration under oblique incidence. These findings can open a door to the design of a broadband MA with multiple MM elements in a single cell configuration.

\section{Acknowledgments}

We gratefully acknowledge the financial support by Scientific Research Projects of Ankara University (BAP) under Grant no. 13B4343015. F.B. also acknowledges "The Scientific and Technological Research Council of Turkey (Tubitak)" through BIDEB-2219 Postdoctoral Research Fellowship.

\section{References}

[1] V.G. Veselago, Soviet Physics Uspekhi 10, 509 (1968).

[2] D.R. Smith, W.J. Padilla, D.C. Vier, S.C. NematNasser, S. Schultz, Phys. Rev. Lett. 84, 4184 (2000).

[3] K.B. Alici, E. Ozbay, Phot. Nano. Fund. Appl. 6, 102 (2008). 
[4] C. Enkrich, M. Wegener, S. Linden, S. Burger, L. Zschiedrich, F. Schmidt, J.F. Zhou, T. Koschny, C.M. Soukoulis, Phys. Rev. Lett. 95, 203901 (2005).

[5] N. Liu, M. Mesch, T. Weiss, M. Hentschel, H. Giessen, Nano Lett. 10, 2342 (2010).

[6] W.L. Chan, H.T. Chen, A.J. Taylor, I. Brenner, M.J. Cich, D.M. Mittleman, Appl. Phys. Lett. 94, 213511 (2009).

[7] P. Singh, K.A. Korolev, M.N. Afsar, S. Sonkusale, Appl. Phys. Lett. 99, 264101 (2011).

[8] H. Oraizi, A. Abdolali, N. Vasegh, Prog. Electromagn. Res. 101, 323 (2010)

[9] D. Schurig, J.J. Mock, D.R. Smith, Appl. Phys. Lett. 88, 041109 (2006).

[10] K.B. Alici, A.B. Turhan, C.M. Soukoulis, E. Ozbay, Opt. Express 19, 14260 (2011).
[11] T.H. Nguyen, S.T. Bui, T.T. Nguyen, T.T. Nguyen, Y. Lee, M.A. Nguyen, D.L. Vu, Adv. Nat. Sci. Nanosci. Nanotechnol. 5, 025013 (2014).

[12] S. Bhattacharyya, S. Ghosh, D. Chaurasiya, K.V. Srivastava, Appl. Phys. A 118, 207 (2015).

[13] F. Ding, Y. Cui, X. Ge, Y. Jin, S. He, Appl. Phys. Lett. 100, 103506 (2012).

[14] H. Tao, C.M. Bingham, D. Pilon, K. Fan, A.C. Strikwerda, D. Shrekenhamer, W.J. Padilla, X. Zhang, R.D. Averitt, J. Phys. D 43, 225102 (2010).

[15] Y.Z. Cheng, Y. Wang, Y. Nie, R.Z. Gong, X. Xiong, X. Wang, J. Appl. Phys. 111, 044902 (2012).

[16] N.I. Landy, S. Sajuyigbe, J.J. Mock, D.R. Smith, W.J. Padilla, Phys. Rev. Lett. 100, 207402 (2008). 